\title{
Investigating the Broadband Microwave Absorption of Nanodiamond Impurities
}

\author{
Jerome A. Cuenca, Evan Thomas, Soumen Mandal, Oliver Williams, and Adrian Porch
}

\begin{abstract}
Broadband microwave complex permittivity measurements of nanodiamond powders are presented. Previous studies show that measurements of dielectric loss strongly correlate with the presence of nondiamond surface impurities. In this study, the frequency dependence of these losses is investigated using the microwave cavity perturbation (MCP) and broadband coaxial probe (BCP) methods. This allowed further understanding as to what mechanisms contribute to the microwave absorption (free electron conduction or dielectric loss from the disordered surfaces). A multimode MCP system is used which utilizes $\mathbf{T M}_{\mathbf{0 n} p}$ modes to provide partial spectral characterization. The MCP results revealed minimal frequency dependence, unlike any static conduction-related mechanism. The BCP measurements corroborate the MCP results with much higher spectral resolution, and further demonstrate that disorder related loss may dominate over free electron conduction from 1-10 GHz. From 0.1-1 GHz, free electron conduction has a greater influence with a characteristic $1 / f$ dependence implying that conduction may dominate at lower frequencies. However, the BCP method, while repeatable, lacks in precision compared to the cavity method. Nonetheless, the major conclusion in this paper is that through simple microwave permittivity measurements, nondiamond carbon impurities in nanodiamond powders are measurable most likely because of disorder related losses as opposed to free electron conduction.
\end{abstract}

Index Terms - Cavity perturbation, coaxial probe, conduction, disorder, nanodiamond, permittivity measurement.

\section{INTRODUCTION}

$\mathbf{N}$ ANODIAMONDS are a new and emerging material showing great practical promise in drug delivery [1]. Studies have shown that administration of chemotherapy agents with nanodiamond drug platforms increases tumor deterioration. The efficacy for these particles is linked to surface termination and functional groups as this determines agglomerate formation and hence the effective surface area for drug adsorption. There are various types of nanodiamond,

Manuscript received June 14, 2015; revised September 26, 2015; accepted October 07, 2015. Date of publication November 17, 2015; date of current version December 02, 2015. The work of J. A. Cuenca was supported in part by Merck KGaA, Darmstadt, Germany and by the U.K. Engineering and Physical Sciences Research Council (EPSRC). This paper is an expanded version from the 2014 Asia-Pacific Microwave Conference, Sendai, Japan, Nov. 4-7, 2014.

J. A. Cuenca and A. Porch are with the School of Engineering, Cardiff University, Cardiff, Wales CF24 3AA, U.K. (e-mail: cuencaj@cardiff.ac.uk; porcha@cardiff.ac.uk).

E. Thomas, S. Mandal and O. Williams are with the School of Physics and Astronomy, Cardiff University, Cardiff, Wales CF24 3AA, U.K. (e-mail: thomase110@cardiff.ac.uk; mandals2@cardiff.ac.uk; williamso@cardiff.ac. uk).

Color versions of one or more of the figures in this paper are available online at http://ieeexplore.ieee.org.

Digital Object Identifier 10.1109/TMTT.2015.2495156 including detonation diamond (DD), diamond produced by high temperature high pressure synthesis (HPHT) and chemical vapor deposition (CVD) each yielding different types and densities of impurities. In this paper we are dealing with HPHT nanodiamonds, where impurities are introduced when a larger diamond is milled; leaving trace amounts of the milling media on the surfaces. Vigorous hydrofluoric and nitric acid treatments are capable of removing many of these impurities [2]. After purification, hydrogen termination can passivate the surface, creating the desired colloidal form for application [3]. Fast and easy examination of the surface purity of nanoparticles becomes paramount for large scale production, which is where we introduce simple microwave characterisation methods.

It is well known that the MCP technique offers a simple measurement of materials used in microwave applications [4]-[6], allowing direct measurement of permittivity and permeability which can be used to assess their performance. The technique has evolved into a means to noninvasively infer conducting properties of materials such as carbon nanotubes [7], superconductors [8] and nanofilms [9]. In this decade microwave measurements are transcending into a noninvasive quality control tool where dielectric and magnetic properties serve as a figure of merit, for example; indirectly inferring moisture content [10], ammonia mass adsorption in zeolites [11] and (as in this paper) nanodiamond purity [12].

In the latter case, it has been demonstrated that MCP offers a much more sensitive quantification of surface amorphous and $\mathrm{sp}^{2}$ carbon when compared to XRD and Raman measurements [13]. From this study we have identified a set of nanodiamond powders with high and low surface impurities. These particles were made using the same synthesis methods but SEM reveals differences in their average sizes; smaller particle sizes contain much higher numbers of impurities since surface impurities scale with surface area.

Preliminary research with MCP was conducted to determine the role of the impurities in the microwave losses [14] and also to determine an optimum characterisation frequency. This investigation inferred that the dielectric loss mechanisms associated with the $\mathrm{sp}^{2}$ and amorphous carbon impurities showed minimal frequency dependence, unlike that due to free electron conductivity which would have diverged as $1 / f$ as frequency $f$ was reduced. This was an interesting find since $\mathrm{sp}^{2}$ carbon is generally associated with high conductivity allotropes such as graphite, carbon nanotubes, and graphene.

However, even though MCP provides a noncontact, high precision measurement at certain frequencies, it greatly sacrifices spectral resolution with a sparse number of measurement frequencies. We must also verify our findings using other methods, 
which is where nonresonant broadband techniques (here using the BCP method) become very valuable.

The major contribution of this study will compare MCP with a much higher resolution (but lower precision) open-ended BCP method. This is to corroborate the finding that microwave absorption in nanodiamond impurities is related to dielectric loss due to the disordered nature of the surfaces as opposed to free electron conductivity normally associated with $\mathrm{sp}^{2}$ carbon. Potentially, this would mean that surface disorder of $\mathrm{sp}^{2}$ and amorphous carbon could be characterized using simple MCP measurements. The BCP method, however, requires contact and is normally used for liquids since homogeneity is then less of an issue, though effective dielectric measurements can still be extracted for powder samples if the packing density adjacent to the flat surface of the probe is kept consistent.

\section{Microwave Techniques}

There are numerous ways to obtain the broadband properties of materials through transmission and reflection based measurement systems including waveguides (air space and dielectric), open-ended transmission lines, free space transmission and planar structures. It is at this point that we stress that the materials under test are powders of low volume. A single waveguide structure is not able to accommodate the multimode range given by the MCP system and it also creates a large sample volume requirement. Free space methods require no contact but careful sample preparation to minimize errors; for example, a flat sample with large volume is needed, which makes this technique unfavorable. This leaves planar and open-ended methods where planar structures generally prevail as they are also capable of measuring permeability [16]. However, since diamond is nonmagnetic, this advantage is not relevant. Though sensitivity is comparable in most senses, fabrication of planar systems is meticulous and the bandwidth is limited to orders of half wavelength structures. The BCP, however, can be easily fabricated from standard microwave connectors and sample preparation is simple.

In this study, we simply wish to verify the broadband results obtained using multiple cavity modes with another broadband technique, for which the BCP will suffice. The notable limitation of both planar and open-ended techniques when measuring powders is ensuring packing consistency in the sample region, which can be somewhat controlled using a sample powder press. We propose to achieve measurement using the BCP method, which is capable of measuring effective dielectric properties in the $0.1-10 \mathrm{GHz}$ range.

\section{BACKGROUND ON PERMitTIVITY}

The complex permittivity of a material occurs due to a combination of harmonic oscillator type polarization mechanisms and when this property is measured as a function of frequency, such mechanisms can be revealed as shown in Fig. 1. Some of the main contributions are due to free electron conductivity, space-charge polarization, permanent dipole relaxation, atomic displacement, and electron cloud displacement.

Fig. 1 can be divided into a low frequency region (conductivity related) and a high frequency region (polarization related). Electron conductivity arises from free electrons or electrons that

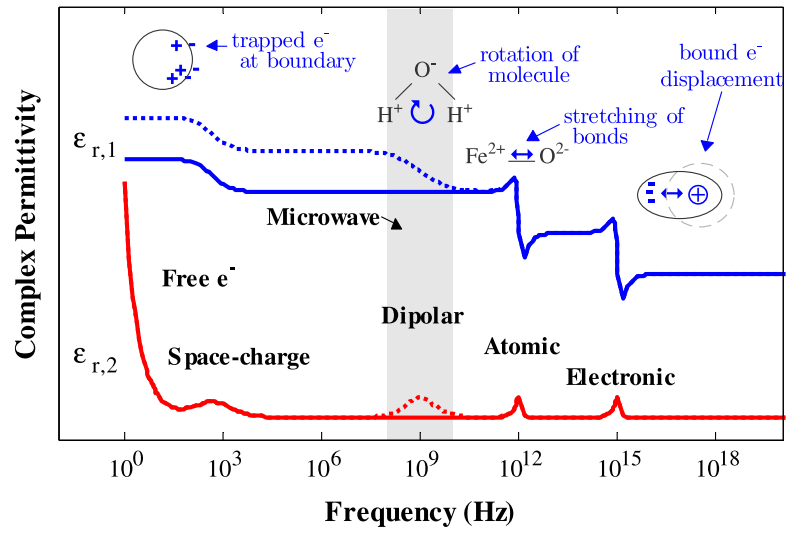

Fig. 1. General overview of the frequency dependent permittivity of a material. In the microwave range, dipolar effects dominate (dotted) but in nonpolar materials, the contribution is mainly due to high frequency polarization related phenomena and decaying low frequency losses [15].

do not participate in bonding in the structure. In pure diamond there are no free electrons and hence any observable conductivity arises due to conducting impurities with nominal contribution from defects in the diamond lattice. For a metallic substance, conductivity is a loss mechanism which results from motion and subsequent scattering of free electrons migrating to the extremities whilst losing energy in collisions with themselves and the bulk lattice. As frequency increases, this effect decreases due to the large effective mass of collections of electrons. Space-charge polarization is essentially the same but results from mixtures of semimetallic materials and dielectrics. On the application of an electric field, the free electrons in this case get trapped at boundaries between the materials, developing a net build-up of charge at the interface. This increases the polarization in the dielectric, causing the overall complex permittivity to increase. It is generally reported in the literature that microwave dielectric losses of graphitic carbon arise due to space charge polarization [17]. For amorphous carbon, multiple scattering and absorption effects from dangling bonds and large disorder can greatly contribute to microwave absorption [18].

At microwave frequencies, dielectric losses may be dominated by friction of permanent dipolar rotation, as in polar solvents such as water. However, in carbonaceous powders no electric permanent dipoles are present. At terahertz frequencies, relaxations of atomic and electronic polarization occur due to the shift from positive charges in nuclei and distortions of electron clouds. These effects will contribute to the polarization of the material (as shown in Fig. 1) but these are fast processes. No such relaxation peaks should be observed at microwave frequencies. The complex permittivity can thus be summarized in the following equation:

$$
\varepsilon_{\mathrm{r}}(\omega)=\varepsilon_{\text {cond }}+\varepsilon_{\text {space }}+\varepsilon_{\text {dipole }}+\varepsilon_{\text {atom }}+\varepsilon_{\text {elec }}
$$

where $\omega$ denotes angular frequency, $\varepsilon_{\mathrm{r}}$ denotes the complex relative permittivity and the subscripts denote contributions from the explained mechanisms.

We have already determined that dielectric loss is far more sensitive for detection of amorphous and $\mathrm{sp}^{2}$ carbon impurities [14]. Since bulk $\mathrm{sp}^{2}$ carbon is a very good conductor it seems 


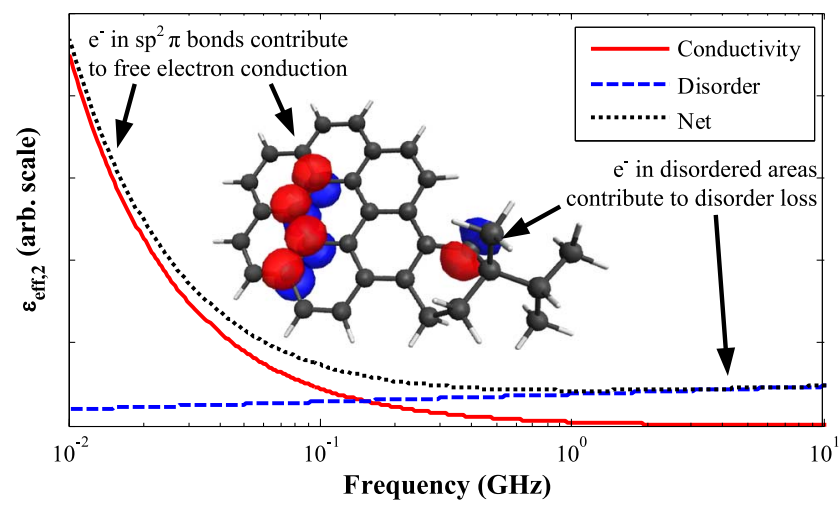

Fig. 2. Simplified contributions to dielectric loss from delocalized electrons (i.e., conductivity and space charge) and polarization related loss mechanisms. Inset shows an abstract representation of the delocalized electrons in the $p$-orbitals in $\mathrm{sp}^{2}$ carbon which gives rise to conductivity and representative disorder created with Avogadro, GAMESS and MacMolPlt [19]-[21].

sensible that there may be conduction loss mechanisms. However, at high frequencies the charge transport can be heavily dependent upon the disordered surfaces as opposed to the free or static conductivity measured at low frequencies [22]. Determination of whether free electron conduction or losses from the disorder plays a role in the microwave absorption can be identified using the model relating relative complex permittivity to conductivity, derived from Maxwell's equations

$$
\varepsilon_{\mathrm{r}, \mathrm{eff}}(\omega)=\varepsilon_{\mathrm{eff}, 1}(\omega)-j\left[\varepsilon_{\mathrm{eff}, 2 \mathrm{~d}}(\omega)+\frac{\sigma_{\mathrm{eff}}(\omega)}{\omega \varepsilon_{0}}\right]
$$

where $\sigma_{\text {eff }}(\omega)$ is the effective free electron conductivity, $\varepsilon_{\text {eff }, 1}(\omega)$ is the effective dielectric constant and $\varepsilon_{\text {eff }, 2 \mathrm{~d}}(\omega)$ is the additional loss contribution related to the disordered surfaces. This is a simplified version of the universal disorder model: $\sigma(\omega) / \sigma_{\text {eff }}=1+\mathrm{k}\left[\omega / \omega_{0}\right]^{\mathrm{s}}$, where in this study the onset frequency $\left(\omega_{0}\right)$ and the fitting parameters $(k$ and $s)$ are simply lumped into $\varepsilon_{\text {eff }, 2 \mathrm{~d}}(\omega)$ [22]. Therefore, free electron conductivity shows a characteristic $1 / f$ dependence. An approximate model for the net contribution to loss is shown in Fig. 2 where at lower frequencies, free electron conductivity dominates and at higher frequencies, disorder related dielectric losses dominate. The frequency dependence of this mechanism is, however, unknown though is normally depicted as relatively constant [22] with an increasing gradient that approaches the relaxation peaks shown at terahertz frequencies in Fig. 1.

\section{Microwave Broadband Coaxial Probe}

The probe consists of an open ended coaxial cable, achieved using an Anritsu K-connector embedded in a brass flange, such that the electric field disperses evanescently at the half-plane at the probe's end, as shown in Fig. 3. Full details of the probe are given in [23]. The sample is simply placed at the end of the probe, where there is a maximum of electric field (owing to the open circuit boundary condition). Complex permittivity can be calculated by measuring the reflection coefficient $\Gamma$ of the probe using the following equation:

$$
\Gamma=\frac{1-Z_{0} Y_{L}}{1+Z_{0} Y_{L}} e^{j \theta}
$$

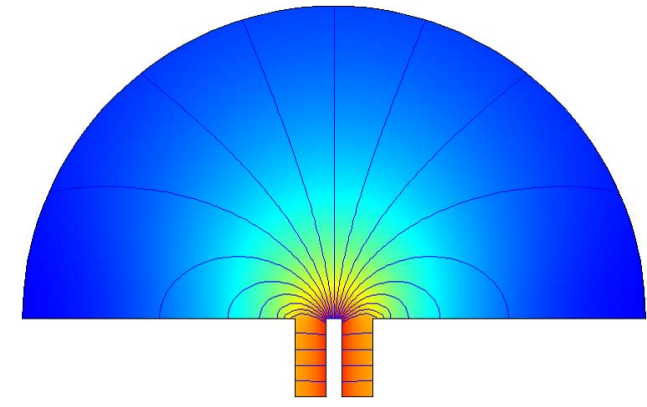

Fig. 3. COMSOL simulation of logarithmic electric field distribution of an open ended coaxial probe in the gigahertz frequency range.

where $Y_{L}$ is the admittance of the end of the probe, $\theta$ is the equivalent phase difference introduced due to the length of the probe and $Z_{0}$ is the impedance of the system $(50 \Omega)$. Calibration to the sample plane can be accomplished using perfect open, short and load terminations for the probe. A simpler approach is to first measure the probe when air-spaced (subscript "a" below) and then measure with a sample load (subscript "L" below), then forming a ratio

$$
\frac{\Gamma_{L}}{\Gamma_{a}}=\frac{1-Z_{0} Y_{L}}{1+Z_{0} Y_{L}} \frac{1+Z_{0} Y_{a}}{1-Z_{0} Y_{a}}
$$

The admittance at the end of the probe can be modelled as two capacitors in parallel giving [23], [24]

$$
Y_{L}=j \omega\left(\varepsilon_{0} \varepsilon_{\mathrm{r}, \mathrm{eff}} C_{0}+C_{f}\right) \approx Y_{a} \varepsilon_{0} \varepsilon_{\mathrm{r}, \mathrm{eff}}
$$

where $C_{0}$ is the capacitance when terminated in free-space, $C_{f}$ is the fringing capacitance and $\varepsilon_{\mathrm{r} \text {,eff }}$ is the effective relative complex permittivity of the powder. The two capacitances can be found by measurement of a known sample (e.g., a block of PTFE) and verified by simulation. The effective permittivity quoted for a powder sample is due to any finite air spaces between the particles, which cannot be removed even with strong compression onto the probe. Hence the calculated value is quoted as being "effective" and so will be smaller than the intrinsic value of $\varepsilon_{\mathrm{r}}$. Equations (4) and (5) can be used to deduce an approximate expression for the complex permittivity valid at lower frequencies [25]

$$
\varepsilon_{\mathrm{r}, \mathrm{eff}} \approx \frac{1}{j \omega \varepsilon_{0} C_{0} Z_{0}}\left(\frac{1-\Gamma_{L} / \Gamma_{a}}{1+\Gamma_{L} / \Gamma_{a}}\right)
$$

\section{Broadband Microwave Cavity Perturbation}

Details of the MCP system and measurements are given in the previous study [14]. The broadband MCP measurements were achieved using a selection of $\mathrm{TM}_{0 n p}$ modes of a cylindrical resonator. The frequencies of these modes can be calculated using the well-known resonant frequency equation of an air-spaced cylindrical cavity

$$
f_{m n p}=\frac{c}{2 \pi} \sqrt{\left(\frac{\alpha_{m n}}{a}\right)^{2}+\left(\frac{p \pi}{l}\right)^{2}}
$$

where $c$ is the speed of light, $a$ and $l$ are the cavity radius and height respectively, $\alpha_{m n}$ is the $n$th root of the $m$ th order Bessel function and $m, n$ and $p$ are integers. The reason why we use 
$\mathrm{TM}_{0 n p}$ modes is due to the fact that the radial 0th order Bessel function has a maximum at $\rho=0$, hence all $\mathrm{TM}_{0 n p}$ modes have a large E-field in the center of the cavity [26]. This means that there will always be a minimum of $\mathrm{H}$-field in the center of the cavity for $\mathrm{TM}_{0 n p}$ modes which is essential for assuming only E-field perturbation. For nanodiamonds this is irrelevant since they are nonmagnetic but as a generic characterisation system for dielectric and magnetic materials, separation of fields is key for solely extracting $\varepsilon_{\mathrm{r}}$. The dimensions of our cavity have been chosen such that the $\mathrm{TM}_{010}$ mode is at approximately $2.5 \mathrm{GHz}$ as this is a common frequency of interest which means selecting $a=46 \mathrm{~mm}$ and $l=40 \mathrm{~mm}$ to reduce interference from TE modes whilst keeping the $\mathrm{TM}_{01 p}$ and $\mathrm{TM}_{02 p}$ modes (for $p \leq 2$ ) below $10 \mathrm{GHz}$.

MCP involves perturbing the fields of these modes and relating the changes in complex frequency to the properties of the material. In this study a low-loss, thin-walled quartz tube containing the powder is placed on the axis of the resonator. The change in frequency due to a small sample perturbation in the E-field of the cavity resonator can be approximated to [27]

$$
-\frac{\Delta \omega}{\omega} \approx \frac{\int_{V_{s}}\left(\varepsilon_{\mathrm{r}, \mathrm{eff}}-1\right) \bar{E}_{s} \cdot \bar{E}_{0}^{*} d V}{2 \int_{V_{c}} \bar{E}_{0} \cdot \bar{E}_{0}^{*} d V}
$$

where $\Delta \omega / \omega$ is the fractional change in complex frequency (real being center frequency and imaginary being half the bandwidth), $V_{\mathrm{s}}$ and $V_{\mathrm{c}}$ are the volumes of the sample and cavity respectively and $\bar{E}_{0}$ and $\bar{E}_{\mathrm{s}}$ are the field vectors in the cavity and in the sample respectively. The complication in this formula is that the absolute fields are generally not known, hence if the condition can be met where $\bar{E}_{\mathrm{s}} \approx \bar{E}_{0}$ then parts of the integrals can be cancelled out. This condition is when the sample is placed in the cavity such that the original field distributions are not grossly altered, or with a minimal depolarization field. This can be achieved by placing a long thin sample parallel to the E-field. The resultant equations for complex permittivity can thus be approximated to [28]

$$
\begin{aligned}
& \varepsilon_{\mathrm{eff}, 1} \approx 2\left(\frac{f_{0}-f_{s}}{f_{0}}\right) \frac{V_{c}}{V_{s}} G_{m n p}+1 \\
& \varepsilon_{\mathrm{eff}, 2} \approx\left(\frac{B W_{s}-B W_{0}}{f_{0}}\right) \frac{V_{c}}{V_{s}} G_{m n p}
\end{aligned}
$$

where $f_{0}$ and $f_{s}$ denote unperturbed and perturbed frequency respectively, $B W_{0}$ and $B W_{s}$ denote unperturbed and perturbed bandwidth respectively, and $G_{m n p}$ is the field filling factor of the cavity dependent upon the sample location and the mode. All values given in (9) and (10) can be measured whereas $G_{m n p}$ can be calculated analytically or through simulation.

\section{A. Analysis of Modes For Broadband Measurement}

The results of COMSOL simulations of the $\mathrm{TM}_{0 n p}$ field distributions are shown in Fig. 4. It is clear that all modes have an E-field along the axis, thus fulfilling the condition of a minimal depolarizing field for a thin sample at the center of the cavity. There are however numerous other modes at frequencies close to the measurement modes. Unfortunately, these are inevitable and it is hard to determine an ideal aspect ratio that separates them. Interference can be mitigated by using coupling structures

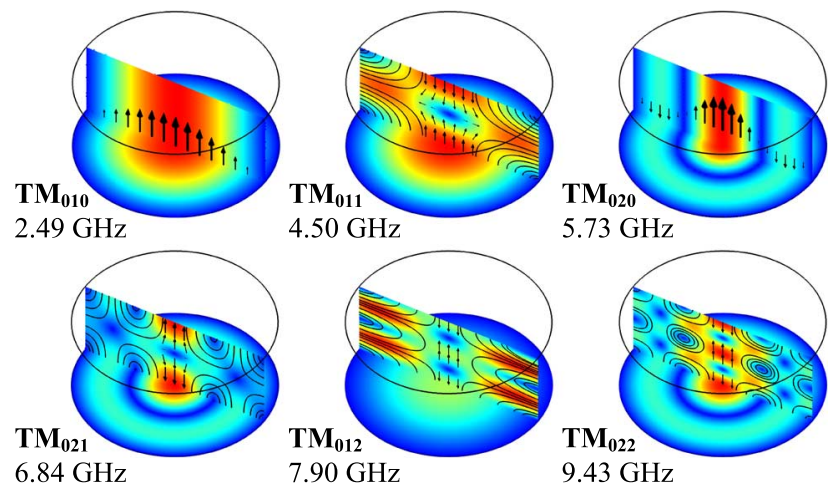

Fig. 4. COMSOL simulation of ideal cylindrical cavity modes used for permittivity measurements. The arrows and the lines denote the direction of the electric field vectors.

that attempt to exclusively excite wanted modes (open circuit coaxial antennas near the center of the top plate prevent excitation of higher order modes propagating around the curved walls). In practice, useful measurement modes can simply be identified by placing high dielectric materials at the sample location and examining the shift in frequency. Additionally, placing a metal rod at the sample location identifies minimally depolarizing modes as these modes should vanish since an E-field cannot exist parallel to a metal surface.

The spatial variation in Fig. 4 will give rise to differing sensitivities to a thin sample placed along the axis. For example $\mathrm{TM}_{0 n 0}$ type modes increase in sensitivity to the sample with increasing $n$ owing to the greater concentration of field on the axis. The sensitivity of a mode is directly related to the field filling factor $G_{m n p}$. It can be calculated by integrating the field intensity $\bar{E}_{\mathrm{s}}^{2}$ and dividing it by the integral of the intensity $\bar{E}_{0}^{2}$ over the cavity volume. These values can be determined analytically but in the previous paper [14] they have been calculated using COMSOL.

Our cavity is not an ideal cylinder and features holes at the top and bottom as well as slightly rounded edges at the corners. It was found through simulation that the rounded edges were responsible for the slightly higher resonant frequencies given in Table I. The $G_{m n p}$ values are also different, with the main cause being due to the sample holes which create distortions in the field distribution at regions of high field. Multiple COMSOL simulations of different hole radii are given in Fig. 5 where the uniform field of the $\mathrm{TM}_{010}$ mode (and consequently other $\mathrm{TM}_{0 n p}$ modes) becomes distorted with increasing hole size. The hole lowers the E-field at the extremities which decreases the field to which the sample is exposed to and, consequently, decreases sensitivity (recall that a lower value of $G_{m n p}$ in Table I means a greater sensitivity to the sample).

The axial hole also affects the resonant frequency and quality factors of the $\mathrm{TM}_{0 n p}$ modes as shown in Fig. 5. The resonant frequency increases with increasing hole length until the length is greater than about $20 \%$ of the cavity length, where no further changes are observed. A large sample hole can give a dramatic reduction in the $\mathrm{Q}$ factor, which is also shown in Fig. 5. For our cavity, the radius of the sample hole is $2.75 \mathrm{~mm}$, which is only $6 \%$ of the cavity radius. The depth is $5 \mathrm{~mm}$ which is $12.5 \%$ of the cavity height. This size of hole therefore has minimal 
TABLE I

RESONANT MODES AND EFFECTIVE SCALING FACTORS

\begin{tabular}{cccccc}
\hline \hline \multirow{2}{*}{$\begin{array}{c}\text { Mode } \\
n p\end{array}$} & \multicolumn{3}{c}{ Frequency $(\mathrm{GHz})$} & \multicolumn{2}{c}{$G_{m n p}$} \\
\cline { 2 - 6 } & Analytical & Simulated & Measured & Analytical & Measured \\
\hline 10 & 2.494 & 2.500 & 2.503 & 0.2695 & 0.2898 \\
11 & 4.502 & 4.521 & 4.532 & 0.8776 & 0.9952 \\
20 & 5.726 & 5.734 & 5.746 & 0.1158 & 0.1247 \\
21 & 6.843 & 6.875 & 6.891 & 0.1654 & 0.1911 \\
12 & 7.899 & 7.931 & 7.946 & 2.7006 & 2.7435 \\
22 & 9.432 & 9.475 & 9.496 & 0.3140 & 0.3448 \\
\hline \hline
\end{tabular}

Simulated and measured are for a cavity with holes and rounded corners. Measured values were obtained with a maximum random error of $5 \mathrm{kHz}$.
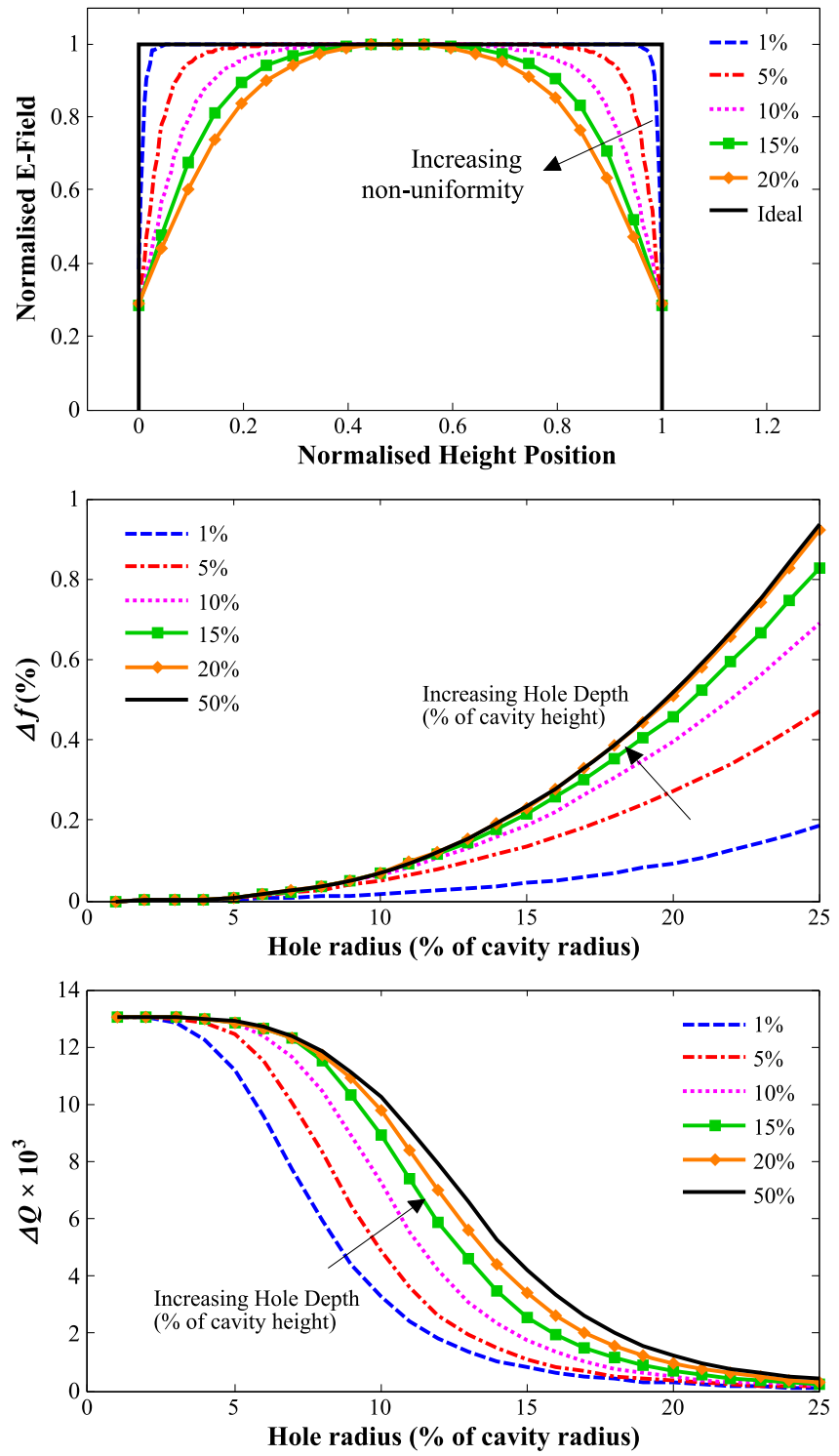

Fig. 5. COMSOL simulation of the effect of the sample hole on the E-field uniformity along the axis of the sample in the $\mathrm{TM}_{010}$ mode (top). The height position is normalized to the cavity height, with 0 and 1 being at the top and bottom, respectively. The plots show different sample hole radii as a percentage of the cavity radius. The effect of hole depth and radius on the frequency (middle) and Q factor (bottom) are also shown. The hole in the COMSOL simulations is modelled as having a scattering boundary condition.

effect both on the frequency and Q factor. The difference in Q factor (shown later in Table II) between the theoretical and the measured, however, is mostly due to the electrical quality of the
TABLE II

Mode SENSITIVITY BASEd ON RANDOM SySTEM ERRORS

\begin{tabular}{|c|c|c|c|c|}
\hline \multirow[b]{2}{*}{$\begin{array}{c}\text { Mode } \\
n p\end{array}$} & \multirow[b]{2}{*}{$\begin{array}{l}\text { Frequency } \\
\quad(\mathrm{GHz})\end{array}$} & \multirow[b]{2}{*}{$\begin{array}{c}\text { Unperturbed } \\
\text { Q Factor }\end{array}$} & \multicolumn{2}{|c|}{ Random Error } \\
\hline & & & $\begin{array}{c}\boldsymbol{\varepsilon}_{\text {eff, } 1} \\
\left(\sigma_{\mathrm{f}}=5 \mathrm{kHz}\right)\end{array}$ & $\begin{array}{c}\boldsymbol{\varepsilon}_{\text {eff, } \mathbf{2}} \\
\left(\sigma_{\mathrm{BW}}=2 \mathrm{kHz}\right)\end{array}$ \\
\hline 10 & 2.503 & $10550 \pm 90$ & $2.5 \times 10^{-3}$ & $4.9 \times 10^{-4}$ \\
\hline 11 & 4.532 & $9120 \pm 40$ & $4.7 \times 10^{-3}$ & $9.4 \times 10^{-4}$ \\
\hline 20 & 5.746 & $15020 \pm 80$ & $4.6 \times 10^{-4}$ & $9.2 \times 10^{-5}$ \\
\hline 21 & 6.891 & $10640 \pm 30$ & $5.9 \times 10^{-4}$ & $1.2 \times 10^{-4}$ \\
\hline 12 & 7.946 & $12020 \pm 40$ & $7.3 \times 10^{-3}$ & $1.5 \times 10^{-3}$ \\
\hline 22 & 9.496 & $12090 \pm 30$ & $7.8 \times 10^{-4}$ & $1.6 \times 10^{-4}$ \\
\hline
\end{tabular}

Unperturbed Q Factor is with the sample tube.

aluminium and the surface roughness inside of the cavity, rather than due to the hole.

Table II shows the sensitivity (or the minimum detectable change due to the random measurement error) of different cavity modes by taking into account the $G_{m n p}$ values and the errors in the measurement of resonant frequency $\left(\sigma_{f}\right)$ and bandwidth $\left(\sigma_{B W}\right)$. Considering mode volume and systematic errors in complex frequency alone, the sensitivity in permittivity measurements is very high and therefore its impact on any uncertainties in the sample measurement is minimized. The variation in sample preparation forms the main source of error in this measurement. To overcome this, multiple samples of the same materials are prepared.

\section{EXPERIMENT}

\section{A. Broadband Coaxial Probe}

The sample is placed at the end of the coaxial flange and is compressed using the structure shown in Fig. 6. A right angled SMA connector and a $3.5 \mathrm{~mm}$ adapter were used to allow the probe to face upright at an appropriate distance while connected to port 1 of a Keysight ENA 5071C. A standard SOLT calibration using an Agilent 85052D calibration kit was carried out to the plane of the $\mathrm{K}$ connector, as shown by the dashed line in Fig. 6. A solid sheet of PTFE was compressed onto the aperture. Assuming the values of $\varepsilon_{\mathrm{r}, 1} \approx 2.1$ and $\varepsilon_{\mathrm{r}, 2} \approx 0.001$ the modelled capacitance of the line $C_{0}$ can be obtained by rearranging (6) which was gave an average of $6.3 \times 10^{-15}$ across the frequency range.

Air measurements were taken for each sample followed by the compressed powder underneath the PTFE plate. Each sample was pressed onto the probe and measured three separate times with standard deviations across the samples obtained at each measured frequency. The stimulus had a 400 point logarithmic sweep between $0.1-10 \mathrm{GHz}$, an IF Bandwidth of 500 $\mathrm{Hz}$, input power of $0 \mathrm{dBm}$ and 32 times averaging. The sample was left on the probe to settle for 10 minutes with averaging restarted prior to the measurement.

To calculate the packing density, the powders were packed into a separate jig with the same compression system displayed in Fig. 6. These samples occupied a cylindrical space with a diameter of $8 \mathrm{~mm}$ and a height of $1 \mathrm{~mm}$. In this configuration, the mass was measured and thus the effective density was extracted. Taking this as a fraction of the material's intrinsic density as given by the datasheet of $3.5 \mathrm{~kg} / \mathrm{cm}^{3}$, the effective packing density can be determined, and this is typically about 0.3 . This 


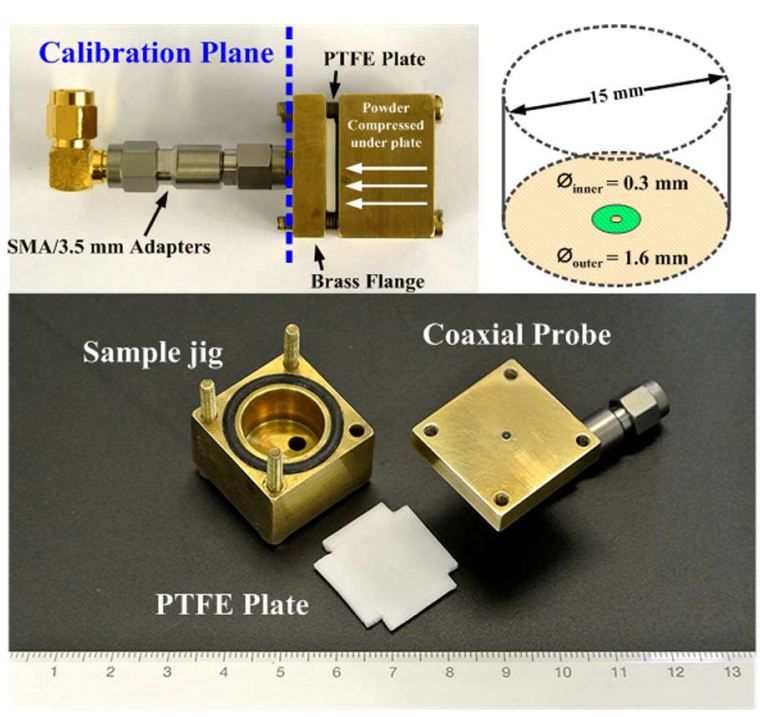

Fig. 6. Photo of the BCP showing the calibration plane and the sample jig (top-left). Dimensions of the aperture and enclosure are given (top-right). Disassembled system (bottom) showing the aperture of the probe (the ruler numerals are every $1 \mathrm{~cm})$. The PTFE sample used for the calibration covered an area of approximately $20 \mathrm{~mm} \times 20 \mathrm{~mm}$ with a thickness of $1.5 \mathrm{~mm}$.



Fig. 7. Photograph of MCP set-up connected to a microwave vector network analyser (VNA) using Huber and Suhner microwave cables. A segmented sweep is shown on the screen to examine the complex frequency response of the $\mathrm{TM}_{0 \mathrm{np}}$ modes. The inset shows the dimensions of the cylindrical cavity.

is important for comparison across the different nanodiamond samples. All of the results are scaled to the intrinsic density of the material in this way.

\section{B. Broadband Microwave Cavity Perturbation}

The MCP measurements were conducted using our aluminium cylindrical cavity (diameter and height of $92 \mathrm{~mm}$ and $40 \mathrm{~mm}$ respectively) as depicted in Fig. 7. As stated previously, the sample hole is $2.75 \mathrm{~mm}$ in diameter, with a small plastic collar outside of the cavity to align the sample tube in the center of the cavity, along its axis. This collar had no effect on the measured resonant frequency and $\mathrm{Q}$ factor since it is well outside of the E-field.
The sample powders were poured into quartz tubes (outer and inner diameters of $2.4 \mathrm{~mm}$ and $2.0 \mathrm{~mm}$ respectively) and left to settle on a vibrating stage for 10 minutes. Quartz was chosen as a tube material as it has very low dielectric loss and thus minimal effect on the unloaded center frequency and bandwidth. The impact of these tubes is that the measurement frequency is brought to a marginally lower value (maximum change across the modes of $13 \mathrm{MHz}$ ) and the initial unperturbed bandwidth is decreased (maximum difference across the modes of $11 \mathrm{kHz}$ ); This latter result is curious, but is due to an increased stored energy due to the polarization of the quartz coupled with its extremely low loss, giving an increased Q factor when it is present. The values for the cavity with the empty quartz tube are used as the unperturbed values given in Table II.

Packing in the tubes was quantified by measuring the mass of the empty and filled tubes whilst taking into account the volume of the filled powder. With mass and volume, the effective density in the tube can be obtained. In a similar manner to the BCP, the quotient of the tube density and the intrinsic density yields the effective packing density.

\section{Sources of Error}

In these measurements, systematic and sample preparation errors are present. For MCP, systematic errors are those associated with the measurement of complex resonance in MCP. The errors in the measurement of complex resonance are small and are given in Table II. The random errors in the sample preparation are expected to dominate in MCP since the tube filling process may settle the powders in different configurations each time.

For BCP, the random systematic errors associated with the reflection coefficient are very low; with averaging enabled, fluctuations in the dielectric constant and losses were less than $5 \%$ of their average value. Calibration of the BCP also contributes to the error. However, this systematic error, associated with $C_{0}$ in the measurement of the PTFE plate, is the same for all samples, and will offset all of the measured values. In addition to this, the random errors in the sample preparation will also contribute. This is through the errors in the measurement of mass and subtle differences in the packing of the powder onto the probe. These factors in particular are the main source of random error.

\section{Samples}

The samples measured in these experiments are the same as those measured in previous studies [13], [14]. These are the Syndia $\mathrm{SYP}_{003}, \mathrm{SYP}_{005}, \mathrm{SYP}_{015}, \mathrm{SYP}_{050}$ and the $\mathrm{SYP}_{125}$ powders provided by Van Moppes Geneva, Switzerland. It has been identified that the $\mathrm{SYP}_{003-015}$ powders have the most nondiamond carbon impurities (with $\mathrm{SYP}_{003}$ as having the highest followed by $\mathrm{SYP}_{005}$ and $\mathrm{SYP}_{015}$ ) while the $\mathrm{SYP}_{050-125}$ powders had no detectable impurities.

\section{DISCUSSION}

\section{A. Comparison of Precision}

The MCP measurements of complex permittivity are presented in Table III with their associated random error bars. It is clear that the random errors in MCP measurements are small for the effective dielectric loss but are large for the 
TABLE III

Calculated Complex Permittivity of Nanodiamond Powders Using MCP

\begin{tabular}{|c|c|c|c|c|c|c|c|c|c|c|c|}
\hline \multirow{2}{*}{ Mode } & \multirow{2}{*}{$\begin{array}{c}\mathrm{f} \\
(\mathrm{GHz})\end{array}$} & \multicolumn{5}{|c|}{ Effective Dielectric Constant $\varepsilon_{\mathrm{eff}, 1}$} & \multicolumn{5}{|c|}{ Effective Dielectric Loss $\varepsilon_{\text {eff,2 }}$} \\
\hline & & $\mathrm{SYP}_{003 \mathrm{C}}$ & $\mathrm{SYP}_{005 \mathrm{C}}$ & $\mathrm{SYP}_{015 \mathrm{C}}$ & $\mathrm{SYP}_{050 \mathrm{C}}$ & $\mathrm{SYP}_{125 \mathrm{C}}$ & $\mathrm{SYP}_{003 \mathrm{C}}$ & $\mathrm{SYP}_{005 \mathrm{C}}$ & $\mathrm{SYP}_{015 \mathrm{C}}$ & $\mathrm{SYP}_{050 \mathrm{C}}$ & $\mathrm{SYP}_{125 \mathrm{C}}$ \\
\hline \multirow[b]{2}{*}{$\mathrm{TM}_{010}$} & \multirow[t]{2}{*}{2.503} & 6.8 & 6.4 & 4.9 & 4.9 & 5.2 & 0.574 & 0.501 & 0.140 & 0.017 & 0.0189 \\
\hline & & \pm 0.3 & \pm 0.1 & \pm 0.1 & \pm 0.2 & \pm 0.1 & $\pm 7 \times 10^{-3}$ & $\pm 2 \times 10^{-4}$ & $\pm 4 \times 10^{-4}$ & $\pm 2 \times 10^{-3}$ & $\pm 2 \times 10^{-4}$ \\
\hline \multirow{2}{*}{$\mathrm{TM}_{011}$} & \multirow{2}{*}{4.532} & 6.7 & 6.3 & 5 & 4.9 & 5.3 & 0.542 & 0.478 & 0.146 & 0.019 & 0.0208 \\
\hline & & \pm 0.2 & \pm 0.1 & \pm 0.2 & \pm 0.1 & \pm 0.1 & $\pm 5 \times 10^{-3}$ & $\pm 1 \times 10^{-3}$ & $\pm 4 \times 10^{-4}$ & $\pm 2 \times 10^{-3}$ & $\pm 3 \times 10^{-4}$ \\
\hline \multirow{2}{*}{$\mathrm{TM}_{020}$} & \multirow[t]{2}{*}{5.746} & 6.8 & 6.3 & 5 & 5 & 5.3 & 0.572 & 0.502 & 0.1525 & 0.016 & 0.0192 \\
\hline & & \pm 0.3 & \pm 0.1 & \pm 0.1 & \pm 0.2 & \pm 0.1 & $\pm 6 \times 10^{-3}$ & $\pm 4 \times 10^{-4}$ & $\pm 2 \times 10^{-5}$ & $\pm 2 \times 10^{-3}$ & $\pm 3 \times 10^{-4}$ \\
\hline \multirow{2}{*}{$\mathrm{TM}_{021}$} & \multirow[t]{2}{*}{6.891} & 6.7 & 6.3 & 5 & 5 & 5.3 & 0.555 & 0.491 & 0.151 & 0.016 & 0.0196 \\
\hline & & \pm 0.2 & \pm 0.2 & \pm 0.2 & \pm 0.2 & \pm 0.1 & $\pm 4 \times 10^{-3}$ & $\pm 2 \times 10^{-3}$ & $\pm 4 \times 10^{-4}$ & $\pm 2 \times 10^{-3}$ & $\pm 4 \times 10^{-4}$ \\
\hline \multirow{2}{*}{$\mathrm{TM}_{012}$} & \multirow[t]{2}{*}{7.946} & 6.6 & 6.3 & 5 & 5 & 5.4 & 0.56 & 0.496 & 0.157 & 0.015 & 0.0191 \\
\hline & & \pm 0.4 & \pm 0.2 & \pm 0.1 & \pm 0.1 & \pm 0.04 & \pm 0.01 & $\pm 4 \times 10^{-3}$ & $\pm 6 \times 10^{-4}$ & $\pm 2 \times 10^{-3}$ & $\pm 2 \times 10^{-4}$ \\
\hline \multirow{2}{*}{$\mathrm{TM}_{022}$} & \multirow[t]{2}{*}{9.496} & 6.5 & 6.2 & 4.9 & 5 & 5.3 & 0.55 & 0.494 & 0.1562 & 0.015 & 0.0196 \\
\hline & & \pm 0.3 & \pm 0.2 & \pm 0.1 & \pm 0.2 & \pm 0.1 & \pm 0.01 & $\pm 4 \times 10^{-3}$ & $\pm 5 \times 10^{-5}$ & $\pm 2 \times 10^{-3}$ & $\pm 5 \times 10^{-4}$ \\
\hline
\end{tabular}

Average values are calculated with error bars being the standard deviation across 3 different tubes of sample. The fractions of the theoretical density for each sample were $0.298,0.298,0.288,0.271$ and 0.298 for $\mathrm{SYP}_{003-125 \mathrm{C}}$ respectively with maximum errors in packing density of $\pm 5 \%$.



Fig. 8. Measured permittivity of nanodiamond samples: real (top) and imaginary (bottom). The lines represent values calculated with the probe method (denoted "P") while the marked points show those achieved with the cavity method (denoted "C"). Shaded regions show the standard deviation obtained across the prepared samples (which includes the measurement of different prepared samples and also the uncertainty in the packing density). All values are normalized to the intrinsic density of $3.5 \mathrm{~kg} / \mathrm{cm}^{3}$ to allow for sample and method comparison.

effective dielectric constant. The impact of this is presented in Fig. 8 where the markers show the MCP data. Clear differences between $\mathrm{SYP}_{003-125}$ can be noticed in the loss measurements, with the error bars being insignificant (smaller than the marker size). The errors for the real parts are likely to be associated with the subtle differences in powder packing between the measurements.

The BCP measurements are the lines and shaded regions in Fig. 8. The random errors are again largest in the effective dielectric constant, to the point that samples are indistinguishable from one another. This is not so much a problem since we have already ascertained from previous studies that the real parts show minimal correlation regarding $\mathrm{sp}^{2}$ and amorphous impurities. The major cause of this was a combination of the calibration and the random sample packing errors. Overall, the random errors are larger for $\mathrm{BCP}$ compared to $\mathrm{MCP}$. Also the errors in between $0.1-1 \mathrm{GHz}$ (further referred to as the low gigahertz range) are much larger than in between $1-10 \mathrm{GHz}$ (further referred to as the high gigahertz range).

The model for the coaxial probe assumes that the material occupies the full, infinite half-space at the end of the probe. The extent to which this field radiates diminishes as materials with larger permittivities are placed at the aperture. In this instance we are probing a low permittivity material with notable differences in loss, but since the real part is low, the evanescent fields may not be contained wholly within the sample. Thus some fields penetrate into the jig and effectively "probe" the sample holder. This causes the measurements of the real part to skew. Since the PTFE plate is a low loss material this does not greatly affect the loss measurements, but at lower frequencies penetration is greater and may radiate further. To avoid this, much larger volumes are required, greater than the jig can support and that are practically available. For the purposes of this measurement system as a sensor for $\mathrm{sp}^{2}$ impurities, this creates a large level of uncertainty for measurements of the real part, which again is not an issue since minimal correlation between impurities and the real part has been found.

\section{B. Frequency Dependent Complex Permittivity}

For the losses, Fig. 8 and Table III show that they are largest in the least pure samples as expected; sample purity was determined in previous studies [13], [14] where $\mathrm{SYP}_{003}, \mathrm{SYP}_{005}$ and $\mathrm{SYP}_{015}$ all had large concentrations of surface $\mathrm{sp}^{2}$ and amorphous carbon and $\mathrm{SYP}_{050}$ and $\mathrm{SYP}_{125}$ had undetectable amounts. The notable contribution in this study is that both the MCP and BCP measurements show similar frequency dependent responses which corroborate the initial findings of disorder related loss mechanisms at microwave frequencies. Moreover, the broader band measurement of BCP reveals significant frequency dependent behavior in the losses in the low gigahertz range and also across the whole range in the real part for $\mathrm{SYP}_{003}, \mathrm{SYP}_{005}$, and $\mathrm{SYP}_{015}$. 
TABLE IV

ESTIMATED CONTRIBUTION TO THE DIELECTRIC LOSS

\begin{tabular}{lcccc}
\hline \hline Sample & $\mathrm{R}^{2}$ on Fit & $\sigma_{\text {eff }}(\mathrm{mS} / \mathrm{m})$ & $\varepsilon_{2, \mathrm{~d}, \mathrm{eff}}$ & $\begin{array}{c}\text { Contribution from } \\
\sigma_{\text {eff }} \text { at } 2.5 \mathrm{GHz}\end{array}$ \\
\hline SYP $_{003 \mathrm{P}}$ & 0.98 & 5.0 & 0.45 & $7 \%$ \\
SYP $_{005 \mathrm{P}}$ & 0.99 & 5.4 & 0.38 & $9 \%$ \\
$\mathrm{SYP}_{015 \mathrm{P}}$ & 0.92 & 1.5 & 0.11 & $9 \%$ \\
$\mathrm{SYP}_{050 \mathrm{P}}$ & 0.97 & 1.0 & 0.02 & $26 \%$ \\
SYP $_{125 \mathrm{P}}$ & 0.91 & 0.4 & 0.005 & $37 \%$ \\
\hline \hline
\end{tabular}

Note: estimation only on the contribution of conductivity to $\varepsilon_{2, \text { eff }}(\omega)$, larger contributions are observed for $\mathrm{SYP}_{050-125}$ implying less loss from the disordered surfaces.

The decrease in loss with frequency in the low gigahertz range is implicit of either free electron conductivity or space-charge polarization, both of which are conduction mechanisms. To establish the potential cause, we must look at the measurements of the real part as well. Free electron conductivity appears in just the losses with a $1 / f$ dependence. Space-charge polarization appears in both the real and imaginary parts of permittivity. Due to the inertia of the movement of large areas of charge, relaxation occurs at low frequencies. This mechanism can therefore be identified by a decrease in both the real and imaginary parts as frequency increases [17]. From Fig. 8, for less pure samples, a negative slope with increasing frequency emerges in both parts, however, the real part continues to decrease even when the losses appear to be frequency independent. Due to this, a clear determination of space charge cannot be reached.

Nevertheless, we can still model the low gigahertz losses as an independent free electron conduction mechanism using the $1 / f$ divergence at low frequencies shown in (2). Taking the average values over the measurement and fitting to this equation, the effective values of conductivity can only be estimated, since the errors in the low frequency region are large. Table IV shows the estimated values for the effective contribution from free electron conductivity and the disorder related dielectric loss mechanisms. The loss mechanism measured with the original MCP method is therefore most likely not caused by free electron conduction and must be some disorder polarization related mechanism. This disordered dielectric loss mechanism has been reported in $\mathrm{sp}^{2}$ carbon allotropes, where these highly conducting materials exhibit an additional loss at gigahertz and terahertz frequencies [22] (the $\varepsilon_{\mathrm{eff}, 2 \mathrm{~d}}$ offset in Table IV). This loss mechanism may be attributed to junctions and defects on the surface where charges are localized [29] which is congruent with losses associated with the disordered nature of the carbon impurities on the nanodiamond; note that disorder related effects may occur from both spurious fractions of amorphous and $\mathrm{sp}^{2}$ carbon as opposed to just the concentration of disorder/amorphous carbon. Interestingly, a larger contribution from $\sigma_{\text {eff }}$ is noticed in $\mathrm{SYP}_{050}$ and $\mathrm{SYP}_{125}$ implying that disorder related effects start to decay with decreasing $\mathrm{sp}^{2}$ concentration. There may even be a threshold of $\mathrm{sp}^{2}$ concentration as to when this starts to dominate. Also, from a quality control perspective, the results show that the high gigahertz range is more appropriate for looking at nanodiamond purity with MCP and $\mathrm{BCP}$ methods due to its approximately constant and consistent response.
For the $\mathrm{SYP}_{050}$ and $\mathrm{SYP}_{125}$ nanodiamond samples, the real part over the entire $0.1-10 \mathrm{GHz}$ range measured here remains constant. This is congruent with electric polarization of species at frequencies much lower than their relaxation frequencies. The difference in values between these and the least pure samples is small which in some ways is expected since all materials are made of carbon atoms, with diamond and carbon black having relatively low dielectric constants [30], [31].

\section{CONCLUSIONS}

In conclusion, we have shown that broadband complex permittivity measurements at microwave frequencies can be achieved using both MCP and BCP methods, with MCP having much higher precision over a broad range. This paper shows that the BCP method corroborates the frequency dependent behavior found with the MCP measurements, in that the dielectric losses of the nanodiamond particles have minimal frequency dependence as opposed to the expected decrease due to delocalized conduction mechanisms on the surfaces of the particles caused by the $\mathrm{sp}^{2}$ carbon fraction. The loss mechanism providing the figure of merit for impurity is hence disorder related, likely to be linked to losses of the disordered nature of the surface $\mathrm{sp}^{2}$ and amorphous carbon impurities, though the exact mechanism has not yet been determined.

\section{ACKNOWLEDGMENT}

Information on the presented data can be found in the Cardiff University data catalogue at http://dx.doi.org/10.17035/d.2015. 100125.

\section{REFERENCES}

[1] E. K. Chow et al., "Nanodiamond therapeutic delivery agents mediate enhanced chemoresistant tumor treatment.," Sci. Transl. Med., vol. 3, no. 73ra21, pp. 1-10, Mar. 2011.

[2] V. Pichot et al., "An efficient purification method for detonation nanodiamonds," Diam. Relat. Mater., vol. 17, no. 1, pp. 13-22, Jan. 2008.

[3] S. Su, J. Li, V. Kundrát, A. M. Abbot, and H. Ye, "Hydrogen-passivated detonation nanodiamond: An impedance spectroscopy study," Diam. Relat. Mater., vol. 24, pp. 49-53, Apr. 2012.

[4] A. Porch, D. Slocombe, and P. P. Edwards, "Microwave absorption in powders of small conducting particles for heating applications.," Phys. Chem. Chem. Phys., vol. 15, no. 8, pp. 2757-2763, Feb. 2013.

[5] I. Kong, S. H. Ahmad, M. H. Abdullah, D. Hui, A. N. Yusoff, and D. Puryanti, "Magnetic and microwave absorbing properties of magnetitethermoplastic natural rubber nanocomposites," J. Magn. Magn. Mater., vol. 322, no. 21, pp. 3401-3409, Nov. 2010.

[6] A. M. Gama, M. C. Rezende, and C. C. Dantas, "Dependence of microwave absorption properties on ferrite volume fraction in $\mathrm{MnZn}$ ferrite/rubber radar absorbing materials," J. Magn. Magn. Mater., vol. 323, no. 22, pp. 2782-2785, Nov. 2011.

[7] A. Porch, D. I. Odili, and P. a Childs, "Microwave characterisation of carbon nanotube powders.," Nanoscale Res. Lett., vol. 7, no. 1, p. 429, Jan. 2012.

[8] A. A. Barannik et al., "Unusual microwave response and bulk conductivity of very thin FeSe0.3Te0.7 films as a function of temperature," Low Temp. Phys., vol. 40, no. 6, pp. 492-499, Jun. 2014.

[9] A. Sulaimalebbe, A. Porch, F. J. Vidal-Iglesias, and G. Attard, "Microwave properties of platinum nanoparticle films," in IEEE MTT-S Int. Microw. Symp. Dig., 2008, vol. 5, no. 2, pp. 1585-1588.

[10] M. Huang, J. Peng, J. Yang, and J. Wang, "Microwave cavity perturbation technique for measuring the moisture content of sulphide minerals concentrates," Miner. Eng., vol. 20, no. 1, pp. 92-94, Jan. 2007.

[11] M. Dietrich, D. Rauch, A. Porch, and R. Moos, “A laboratory test setup for in situ measurements of the dielectric properties of catalyst powder samples under reaction conditions by microwave cavity perturbation: Set up and initial tests.," Sensors (Basel)., vol. 14, no. 9, pp. 16856-68, Jan. 2014. 
[12] D. Slocombe, A. Porch, E. Bustarret, and O. Williams, "Microwave properties of nanodiamond particles," Appl. Phys. Lett., vol. 102, no. 24, p. 244102, Jun. 2013.

[13] J. A. Cuenca, E. Thomas, S. Mandal, O. Williams, and A. Porch, "Microwave determination of sp2 carbon fraction in nanodiamond powders," Carbon J., vol. 81, pp. 174-178, Jan. 2015.

[14] J. A. Cuenca, E. Thomas, S. Mandal, O. Williams, and A. Porch, "Broadband microwave measurements of nanodiamond," in Proc. Microw. Conf. (APMC), 2014 Asia-Pac., 2014, pp. 441-443.

[15] T. Prodromakis and C. Papavassiliou, "Engineering the MaxwellWagner polarization effect," Appl. Surf. Sci., vol. 255, no. 15, pp. 6989-6994, May 2009.

[16] B. Kang, J. Cho, C. Cheon, and Y. Kwon, "Nondestructive measurement of complex permittivity and permeability using multilayered coplanar waveguide structures," IEEE Microw. Wirel. Compon. Lett., vol. 15 , no. 5, pp. 381-383, May 2005.

[17] J. Kim et al., "Flexible and transparent dielectric film with a high dielectric constant using chemical vapor deposition-grown graphene interlayer," ACS Nano, vol. 8, no. 1, pp. 269-274, Dec. 2014.

[18] T. Zhao et al., "Electromagnetic wave absorbing properties of amorphous carbon nanotubes.," Sci. Rep., vol. 4, no. 5619, pp. 1-7, Jan. 2014.

[19] M. D. Hanwell, D. E. Curtis, D. C. Lonie, T. Vandermeersch, E. Zurek, and G. R. Hutchison, "Avogadro: An advanced semantic chemical editor, visualization, analysis platform.," J. Cheminform., vol. 4, no. 1, p. 17, Jan. 2012

[20] M. S. Gordon and M. W. Schmidt, "Advances in electronic structure theory: GAMESS a decade later," in Theory and Applications of Computational Chemistry: The First Forty Years. New York, NY, USA Elsevier, 2005, pp. 1167-1189.

[21] B. M. Bode and M. S. Gordon, "Macmolplt: A graphical user interface for GAMESS," J. Mol. Graph. Model., vol. 16, no. 3, pp. 133-138, Jun. 1998.

[22] J. S. Bulmer et al., "Microwave conductivity of sorted CNT assemblies.," Sci. Rep., vol. 4, no. 3762, pp. 1-8, Jan. 2014.

[23] A. Porch et al., "Microwave treatment in oil refining," Appl. Petrochem. Res., vol. 2, no. 1-2, pp. 37-44, Aug. 2012.

[24] S. Evans and A. B. Azeman, "Radiofrequency and microwave dielectric properties of aqueous cryoprotectant agents: Dimethyl sulphoxide and 2,3-butanediol," Phys. Med. Biol., vol. 43, no. 10, pp. 2817-2829, Oct. 1998.

[25] C. Gabriel, T. Y. A. Chan, and E. H. Grant, "Admittance models for open ended coaxial probes and their place in dielectric spectroscopy," Phys. Med. Biol., vol. 39, no. 12, pp. 2183-2220, Dec. 1994.

[26] D. M. Pozar, Microwave Engineering. Hoboken, NJ, USA: Wiley, 2004.

[27] U. Raveendranath and K. T. Mathew, "New cavity perturbation technique for measuring complex permeability of ferrite materials," $M i$ crow. Opt. Technol. Lett., vol. 18, no. 4, pp. 241-243, Jul. 1998.

[28] M. Lin and M. N. Afsar, "A new cavity perturbation technique for accurate measurement of dielectric parameters," in IEEE MTT-S Int. Microw. Symp. Dig., 2006, pp. 1630-1633.

[29] Y. Wang, M. Jasiswal, M. Lin, S. Saha, B. Ozyilmaz, and K. P. Loh, "Electronic properties and applications of nanodiamond," ACS Nano, vol. 6 , no. 2, pp. 1018-1025, Feb. 2012.

[30] M. Hotta, M. Hayashi, M. T. Lanagan, D. K. Agrawal, and K. Nagata, "Complex permittivity of graphite, carbon black and coal powders in the ranges of X-band frequencies $(8.2$ to $12.4 \mathrm{GHz})$ and between 1 and $10 \mathrm{GHz}, "$ ISIJ Int., vol. 51, no. 11, pp. 1766-1772, Nov. 2011.

[31] S. Heidger, S. Fries-Carr, J. Weimer, B. Jordan, and R. Wu, "Dielectric characterization of microwave assisted chemically vapor deposited diamond," in Proc. IEEE Nat. Aerosp. Electron. Conf. (NAECON'98), 1998, pp. 247-254.

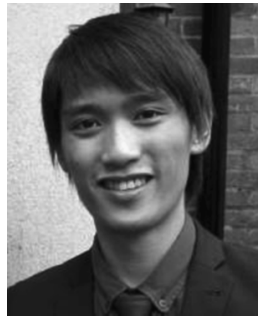

Jerome A. Cuenca received the B.Eng. in electronic and communications engineering from Cardiff University, Wales, U.K., in 2012. He is currently working toward the Ph.D. degree at the Centre for High Frequency Engineering, Cardiff University.

His work focuses on using microwave measurement systems in the field of materials science with keen interests in carbon and iron oxides.

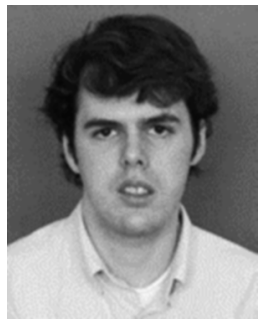

Evan Thomas received the M.Eng. degree in electronic engineering with communications engineering from the University College London, London, U.K., in 2012, and is currently working towards the Ph.D. degree at Cardiff University, Wales, U.K.

His research focuses on the processing and characterisation of nanocrystalline diamond films and particles, with particular interest in the use of diamond for microelectro-mechanical systems (MEMS).

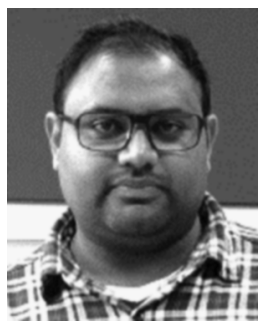

Soumen Mandal received the M.Sc. and Ph.D. degrees in physics from IIT Kanpur, Kanpur, India, in 2008.

$\mathrm{He}$ is a Research Associate with the School of Physics and Astronomy, Cardiff University, Wales, U.K. His research mainly focuses on devices made from boron doped diamond and characterization of diamond particles for variety of applications.

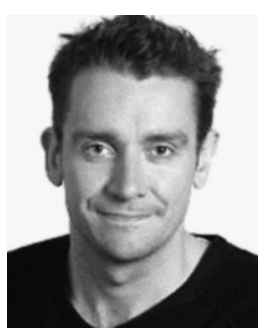

Oliver Williams received the B. Eng. and Ph.D. degrees in electronic and electrical engineering from University College London, London, U.K., in 2003.

$\mathrm{He}$ is currently Professor of Experimental Physics at Cardiff University, Wales, U.K., and head of the Cardiff Diamond Foundry. Previously, he was Head of diamond technology at the Fraunhofer Institute for Applied Physics. He has over 15 years of experience in diamond growth and technology. He is currently developing custom diamond nanoparticles with custom color centers as well as superconducting

diamond devices.



Adrian Porch received the M.A. degree in physics and the Ph.D. degree in low temperature physics from Cambridge University, Cambridge, U.K., in 1989.

$\mathrm{He}$ is a Professor with the School of Engineering, Cardiff University, Wales, U.K., and a member of the Centre for High Frequency Engineering. He has 30 years of experience in applying microwave methods to measure and understand the fundamental properties of electronic materials. More recently, his techniques have been used to develop new types of electromagnetic sensors, with emphasis on applications 